\title{
Osteogenic capacity of nanocrystalline bone cement in a weight-bearing defect at the ovine tibial metaphysis
}

This article was published in the following Dove Press journal:

International Journal of Nanomedicine

14 June 2012

Number of times this article has been viewed

\author{
Christoph Harms' \\ Kai Helms' \\ Tibor Taschner' \\ loannis Stratos' \\ Anita Ignatius ${ }^{2}$ \\ Thomas Gerber ${ }^{3}$ \\ Solvig Lenz ${ }^{4}$ \\ Stefan Rammelt ${ }^{5}$ \\ Brigitte Vollmar ${ }^{6}$ \\ Thomas Mittlmeier ${ }^{1}$ \\ 'Department of Trauma and \\ Reconstructive Surgery, University \\ of Rostock, Rostock, 'Institute \\ of Orthopaedic Research and \\ Biomechanics, University of Ulm, \\ Ulm, ${ }^{3}$ Department for Materials \\ Research and Nanostructures, \\ Institute for Physics, ${ }^{4}$ Department of \\ Oral and Maxillofacial Plastic Surgery, \\ University of Rostock, Rostock, \\ ${ }^{5} \mathrm{Clinic}$ of Trauma and Reconstructive \\ Surgery, University Hospital "Carl \\ Gustav Carus", Dresden, 'Institute for \\ Experimental Surgery, University of \\ Rostock, Rostock, Germany
}

Correspondence: Christoph Harms Department of Trauma and Reconstructive Surgery, University of Rostock, Schillingallee 35, D 18057 Rostock, Germany Email christoph.harms@med.uni-rostock.de

\begin{abstract}
The synthetic material Nanobone ${ }^{\circledR}$ (hydroxyapatite nanocrystallines embedded in a porous silica gel matrix) was examined in vivo using a standardized bone defect model in the ovine tibial metaphysis. A standardized $6 \times 12 \times 24-\mathrm{mm}$ bone defect was created below the articular surface of the medial tibia condyles on both hind legs of 18 adult sheep. The defect on the right side was filled with Nanobone ${ }^{\circledR}$, while the defect on the contralateral side was left empty. The tibial heads of six sheep were analyzed after 6,12 , and 26 weeks each. The histological and radiological analysis of the defect on the control side did not reveal any bone formation after the total of 26 weeks. In contrast, the microcomputed tomography analysis of the defect filled with Nanobone ${ }^{\circledR}$ showed a $55 \%, 72 \%$, and $74 \%$ volume fraction of structures with bone density after 6, 12, and 26 weeks, respectively. Quantitative histomorphological analysis after 6, and 12 weeks revealed an osteoneogenesis of $22 \%$, and $36 \%$, respectively. Hematoxylin and eosin sections demonstrated multinucleated giant cells on the surface of the biomaterial and resorption lacunae, indicating osteoclastic resorptive activity. Nanobone ${ }^{\circledR}$ appears to be a highly potent bone substitute material with osteoconductive properties in a loaded large animal defect model, supporting the potential use of Nanobone ${ }^{\circledR}$ also in humans.
\end{abstract}

Keywords: biocompatibility, bone ingrowth, hydroxyapatite, osseointegration, osteoconduction

\section{Introduction}

The treatment of defects in large bones is a challenge for orthopedic surgeons. Bone transplants or bone substitutes are necessary in about $10 \%$ of all reconstructive procedures due to trauma or to fill resectional voids as well as to correct congenital defects. Autologous bone grafting continues to be the "gold standard" for defect filling in reconstructive surgery. Nevertheless, major problems still exist with autologous bone grafting. These include donor site morbidity with residual complications in up to $50 \%$ of the procedures and the limited availability of autologous bone itself. ${ }^{1}$ Allografts have been used extensively but these raise concerns of potential contamination and mechanical stability. Sensitive blood tests, donor screening, and extensive efforts to clean bone and clear it of infectious agents have helped to alleviate the concern regarding contamination. Fatigue fractures are a common problem when using cortical allografts. Many of the failures of these bone grafts are due to mechanical problems that are related to the biologic incorporation of the graft. Inorganic implants, such as synthetic calcium-phosphate-based bone substitutes, seem to be useful alternatives to autologous bone grafting. ${ }^{2}$ Hydroxyapatite (HA) is the main inorganic bone matrix component. Synthetic HA, when used as a bone graft, has shown a high degree of biocompatibility and support for cellular activity. It stimulates osteoconduction 
and is generally slowly replaced by the host bone after implantation. ${ }^{3}$ Since the 1980 s, blocks and granules of porous synthetic HA have been used in orthopedic, craniofacial, and dental applications. ${ }^{4}$ Continuous improvements in synthesis, manufacturing technology, and purification have led to a variety of synthetic HA materials and forms with different properties. ${ }^{1,5}$

Broadly termed "bioceramics," these forms of synthetic HA and beta-tricalciumphosphate ( $\beta$ TCP) are increasingly being used as bone substitutes for grafting into defects or as coatings on permanent implants. The bone graft substitute Nanobone ${ }^{\circledR}$ used in this study is a nanocrystalline hydroxyapatite embedded in a porous silica gel matrix. Unlike conventional HA forms that are usually sintered at temperatures ranging from $700^{\circ} \mathrm{C}$ up to $1200^{\circ} \mathrm{C}$, Nanobone ${ }^{\circledR}$ is produced at a temperature $<200^{\circ} \mathrm{C}$. ${ }^{6}$ The lower processing temperature has a profound effect on the material's porosity and surface area. For example, a large surface area enhances the solubility, tissue reaction, and osteoclastic activity.

Nanobone ${ }^{\circledR}$ has been shown to have in vivo osteoconductive properties on dermal bone. ${ }^{7}$ The material has been tested in jaws of mini-pigs and has recently been used successfully to fill dentoalveolar defects in man. ${ }^{8-10}$ This early clinical experience with the material in mandibular reconstructive surgery has been encouraging. However, there is no experimental or clinical experience with the use of Nanobone ${ }^{\circledR}$ in a weight-bearing long-bone model. The aim of this investigation was to assess the biodegradation and the osteogenic potential of this nanocrystalline HA-based bone grafting material in a standardized weight-bearing defect in sheep. The sheep model is often used in biomaterial testing due to a higher similarity of the sheep's bone metabolism to the human situation as compared to the bone metabolism of small animals.

\section{Methods}

The bone replacement material Nanobone ${ }^{\circledR}$ (Artoss, Rostock, Germany) used in this study is a synthetic HA scaffold produced in a sol-gel process at temperatures of $<200^{\circ} \mathrm{C}$. ${ }^{6}$ The material consists of nanocrystalline HA embedded in a silica gel matrix. Solvent evaporation leads to the formation of small pores $(\varnothing 5-100 \mu \mathrm{m})$ in the structure. After solvent evaporation the HA crystallites are loosely packed and held together by silica $\left(\mathrm{SiO}_{2}\right)$. Because of the interconnecting pores the specific surface area of the material is high on the order of $84 \mathrm{~m}^{2} / \mathrm{g}$. The volume fraction of the granules is about $61 \%$. The granules have a density of $0.4-0.7 \mathrm{~g} / \mathrm{cm}^{3}$ and a typical compressive strength on the order of $40 \mathrm{MPa}{ }^{11}$
Low temperature processing of the material offers several advantages. ${ }^{12}$ The rough surface structure of the granular material is a result of the material's interconnecting porous structure. The porous structure and optimum pore size allow and promote bone ingrowth. Histological and immunohistochemical studies demonstrated its osteoconductive nature and early remodeling in the host tissue. ${ }^{10,13,14}$ Animal experiments, using a mini-pig critical-size defect model in alveolar bone, showed high rates of bone formation and nearly complete resorption of the implant within 8 months. ${ }^{15}$

\section{Animal model}

The study protocol of this investigation followed national guidelines and regulations for the care and use of laboratory animals and was approved by the local authorities (Landesamt für Landwirtschaft, Lebensmittelsicherheit und Fischerei Mecklenburg-Vorpommern, Germany; LALLF M-V/TST/7221.3-1.1-019/06).

A well-established, weight-bearing, large animal model ${ }^{16-18}$ was used for investigation of the graft material. Eighteen mature female Mecklenburg sheep with an average body weight of $63 \mathrm{~kg}$ were operated on under general anesthesia induced by intravenous injection of propofol. The sheep were maintained with isoflurane in $100 \%$ oxygen. Continuous electrocardiogram and pulsoxymetry were used during the operation to monitor the sheep. Novaminsulfon was given perioperatively for pain relief and was continued for 2 days after the procedure. Antibiotic prophylaxis consisted of amoxicillin administered intravenously as a single dose preoperatively. The sheep were placed in supine position and both legs were shaved and disinfected with povidone-iodine. A longitudinal incision $15 \mathrm{~cm}$ in length was made on the medial side of the knee to expose the proximal tibia. Under continuous cooling with saline, a wedgeshaped bone defect of $6 \times 12 \times 24 \mathrm{~mm}$ was cut in the medial aspect of the left and right proximal tibia $3 \mathrm{~mm}$ beneath the articular surface, using a specially designed guiding fixture. ${ }^{16}$ Bone debris was removed by lavage, and bleeding was stopped by tamponade. On the left side the defect was left empty. On the right side the defect was filled with the bone substitute, which had been mixed with $5 \mathrm{~mL}$ of venous blood according to the manufacturer's instructions. The wound was closed in layers with absorbable sutures and covered with a dressing. Subsequently, the sheep were returned to their stable. Due to the integrity of the lateral condyles and the dense bone structure in sheep, no additional stabilization was needed. The animals were mobilized under full weight bearing of both hind legs.

At intervals of 6,12 , and 26 weeks after the intervention, six sheep, in deep anesthesia induced with pentobarbital, 
were sacrificed at each time point with potassium chloride. The operated tibiae were harvested and evaluated for periosteal integrity. Conventional radiographs and computed tomography (CT) scans were performed. The conventional CT scans were analyzed with a DICOM viewer (Osirix 3.5; Osirix Imaging Software, Geneva, Switzerland).

\section{Micro-CT}

After the specimens were harvested, a micro-CT was performed on a bone block of $30 \times 50 \times 60 \mathrm{~mm}$ removed from the tibia. Six models with the same-sized defect as the in vivo defect were fabricated from a rigid plastic and filled with Nanobone ${ }^{\circledR}$ in the same manner as was done in the reference sheep.

\section{Histological and histomorphometric evaluation}

After fixation in formalin the harvested specimens were decalcified in neutral 10\% ethylene diamine tetraacetic acid (EDTA) for 14 days, processed with graded concentrations of alcohol and embedded in paraffin. Decalcified sections were processed and stained with hematoxylin and eosin (HE) and Goldner-Masson trichrome to morphologically visualize the new bone formation and to distinguish between mature and immature bone. Enzyme and immunohistochemical analysis was performed with the respective markers as described previously. ${ }^{19,20}$ Enzyme histochemical staining against tartrate-resistant acid phosphatase (TRAP) specifically stains osteoclasts and mononuclear precursor cells, indicating bone resorption during the remodeling process around and within the bone substitute. Osteocalcin as a noncollagenous matrix protein is expressed by osteoblasts and is localized in the cement lines of newly formed bone trabeculae. Without background staining it is specifically seen in mature osteoblasts.

Semiquantitative histomorphometric analysis was performed on micrographs from the HE-stained sections at $1.25 \times$ magnification using planimetric analysis (Axio Vision LE; Zeiss, Jena, Germany). The slices were scanned (Agfa Horizon Ultra $^{\circledR}$; Agfa, Köln, Germany) for documentation and for histomorphometric evaluation. The original images were prepared for histomorphometric analysis by editing with Adobe Photoshop ${ }^{\circledR}$ software (v. 6.0; Adobe, San Jose, CA). The implantation site where Nanobone ${ }^{\circledR}$ granules could be localized was defined as a region of interest (ROI). This area was digitally marked and measured using the AnaLYSIS ${ }^{\circledR}$ software (Soft Imaging System; GmbH, Münster, Germany). Within the ROI three fractions of tissue could be differentiated: newly formed bone, remnant bone graft substitute, and connective soft tissue. The areas with newly formed bone and with the remnant bone graft substitute were marked with different colors and the dimensions of these areas were determined. By subtracting these areas from the ROI, the area of soft tissue was calculated, and by relating these measured and calculated areas to the ROI, the percentage area for each could be established. The percentage of new bone that had been formed within the defect was calculated as well as the percentage of remaining bone graft material. The bone per tissue volume and the remaining graft material per tissue volume were determined, and the fraction of soft tissue was calculated. This process was repeated in three different sections of the same defect to ensure a representative sampling over the entire defect.

\section{Statistical analysis}

All data were expressed as means \pm standard error of the mean. After testing for normality and equal variance across groups, difference points were assessed by two-way analysis of variance followed by an appropriate post-hoc comparison. For reasons of clarity and comprehensiveness, only statistical significant differences between the groups are shown in the figures. Statistical significance was set at $P<0.05$. Statistics were performed using the GraphPad Prism $5^{\circledR}$ software package (GraphPad Software, San Diego, CA).

\section{Results}

The animals returned to a normal walking pattern within 7 days. No relevant complications necessitating surgical revision or sacrifice of the animals occurred throughout the experimental period.

The morphological investigation of the harvested tibiae showed an intact joint surface. The medial surface of the right and left tibiae was covered with a thick layer of scar tissue. Macroscopically, no sign of inflammation or adverse tissue reaction was seen.

Radiographic examination of the tibiae harvested from the left side (control) over the 26-week experimental period did not show spontaneous osseous ingrowth in any of the sheep. Conventional CT scanning confirmed the findings obtained by standard X-rays. Within the defect on the left side, no radiopaque tissue could be observed. By measuring the frontal CT reconstruction, the extent of the defect was shown to have remained constant at $6 \mathrm{~mm}$. The control defect was filled with nonspecific fibrous tissue after 6, 12, and 26 weeks as seen in Figure 1A. Histological analysis did not show any bone formation within the left tibia (Figure 1B). 

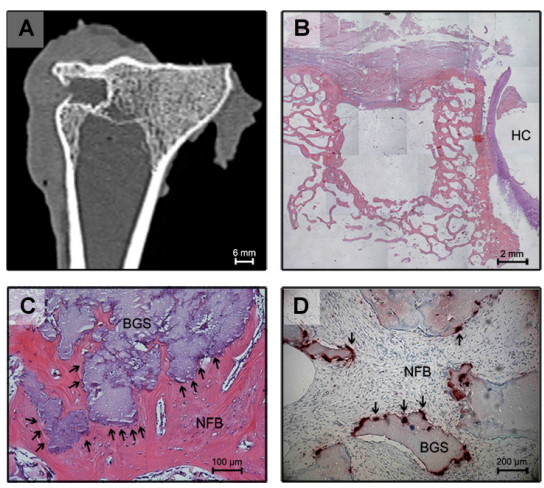

Figure I Frontal reconstruction of conventional computed tomography of the left tibia 6 months after creation of the defect. Bulky scar tissue was observed at the outer side of the defect. The defect cavity was filled with fibrous connective tissue (A). Histological section of the defect in the left tibia 6 months after creation of the defect $(\mathrm{HE}$, original magnification $25 \times)$ : There is no bony ingrowth into the defect. The defect itself is empty because the loose-fitting interconnecting tissue washed out during histological preparation. Hyaline cartilage can be seen on the right side (B). Histological section 6 weeks after implantation of the bone substitute material (HE, original magnification (00x): The implant is completely embedded into new bone. At the surface of the biomaterial, multiple darkly stained giant cells are detectable, marked by arrowheads (C). Histological section 6 weeks after implantation (TRAP staining, original magnification 200x): Arrowheads indicate TRAP-positive multinucleated cells adjacent to the biomaterial at the interface (D).

Abbreviations: $\mathrm{HE}$, hematoxylin and eosin; $\mathrm{HC}$, hyaline cartilage; NFB, newly formed bone; BGS, bone graft substitute; SCT, soft connective tissue; TRAP, tartrate-resistant acid phosphatase.

The connective tissue that had formed within the defect was so loosely adherent that it was lost during sample preparation and could not be further evaluated histologically.

With histological and immunohistochemical analysis of the right tibiae, new bone formation and neovascularization were observed around the hydroxyapatite particles within the defect. Light microscopic evaluation revealed a consistent bone response within each of the six specimens at each time period.

After 6 weeks multiple multinucleated giant cells and macrophages were seen at the interface of bone and the HA (Figure 1C). Judging from their enzyme histochemical activity against TRAP, these cells appear to be osteoclasts (Figure 1D).

An increase in the progression of new bone formation within the bone substitute material continued from 6 to 12 weeks. Bone ingrowth occurred primarily from the margins of the defect toward the center of the defect. Newly formed bone was noted adjacent to the HA material (Figure 2A and B). Immunohistochemical staining against osteocalcin showed layers of osteoblasts depositing bone directly on the implant surfaces (Figure 2C).

At the end of the observation period, 6 months after implantation, the cells on the surface of the newly formed bone had the appearance of bone-lining cells. Fibrous encapsulation of the granules was not noted at any time and osteocytes were
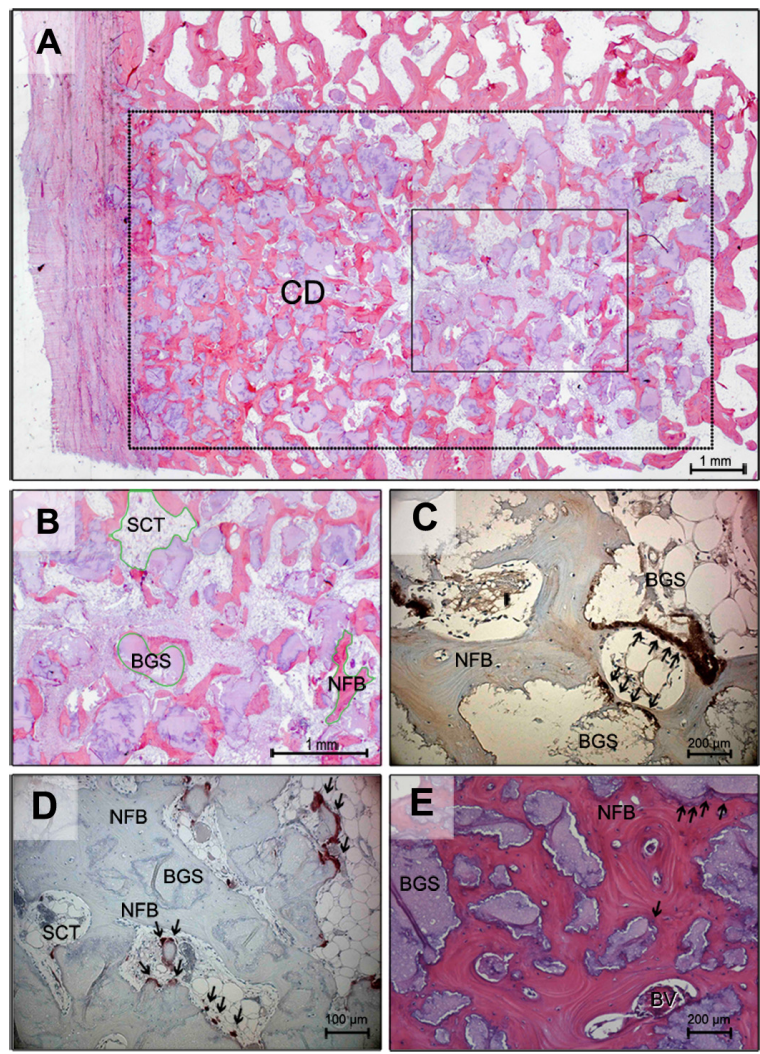

Figure 2 Histological section 12 weeks after implantation (HE, original magnification $25 \times)$ : ROI is marked by a dotted line. Bony ingrowth occurs from the margins of the defect, especially from the periost to the center of the defect (A). Detail of Figure IA: The fractions of tissue are marked (B). Histological section 26 weeks after implantation (osteocalcin staining, 200x): Osteoblast activity along the biomaterial and fibrous tissue is visualized (arrowheads). Cement lines within the newly formed bone also stain positively for osteocalcin (C). Histological section 26 weeks after implantation (TRAP staining, original magnification 100x): The granules of the biomaterial are mostly surrounded by new bone formation, but in contrast to fibrous tissue osteoclasts are still detectable (marked by arrowheads) (D). Histological section 26 weeks after implantation ( $\mathrm{HE}$, original magnification I00x): After a halfyear, cellular activity is still apparent at the interface between bone and biomaterial, particularly near the newly formed blood vessels (E).

Abbreviations: $\mathrm{HE}$, hematoxylin and eosin; CD, center of the defect; NFB, newly formed bone; BGS, bone graft substitute; ROI, region of interest; SCT, soft connective tissue; BV, blood vessel; TRAP, tartrate-resistant acid phosphatase.

found in close proximity to the surfaces of the Nanobone ${ }^{\circledR}$ implant. The surfaces of the implanted material were entirely covered with bone and the granules of Nanobone ${ }^{\circledR}$ were fully integrated into newly formed bone. The majority of surfaces were populated with cuboidal darkly stained cells, indicating active osteoblasts. The TRAP-positive multinucleated cells were also observed after 26 weeks within the new bone and in large numbers adjacent to the granules, indicating continuous remodeling (Figure 2D). Neovascularization was also evident within the pores at this time point (Figure 2E).

Histomorphometric analysis established that the majority of bone ingrowth occurred within the first 6 weeks and was terminated within 3 months. After 6 weeks $22 \%$ of the ROI was filled with bone, and after 3 months this proportion had increased 
to $36 \%$. This increase was statistically significant. Within the following 3 months, there was no further increase of bone ingrowth. After 6 months the volume fraction of bone was found to be approximately $30 \%$. This difference was not statistically significant. The proportions of bone, bone grafting substitute, and connective soft tissue remained constant (Figure 3).

Micro-CT measurement was limited by the fact that the bone substitute material had a very similar X-ray adsorption to bone. Therefore, bone and bone substitute material could not be differentiated and were both set in relation to the unfilled space. Similar to the histomorphometric findings, a continuous decrease of soft tissue and a significant increase of radiopaque material with a bone-like appearance could be detected within the first 12 weeks. This bone-like material accounted for $55 \%$ of the defect volume after 6 weeks and increased to $73 \%$ and $78 \%$ after 3 months and 6 months, respectively (Figure 4).

Radiographic evaluation of the right tibiae filled with the bone substitute showed an increasing level of radiopacity both in standard radiographs and in conventional CT scans. In particular, the latter showed the defect area being filled with a high density material following 6,12 , and 26 weeks (Figure 4A-C).

\section{Discussion}

The ideal artificial bone substitute requires good biocompatibility without inflammation or toxicity, sufficient mechanical strength, strong bonding to the host bone, and active osteoconductivity to support bone ingrowth into the graft. Ultimately, the material would be replaced by or incorporated into host bone..$^{21,22}$

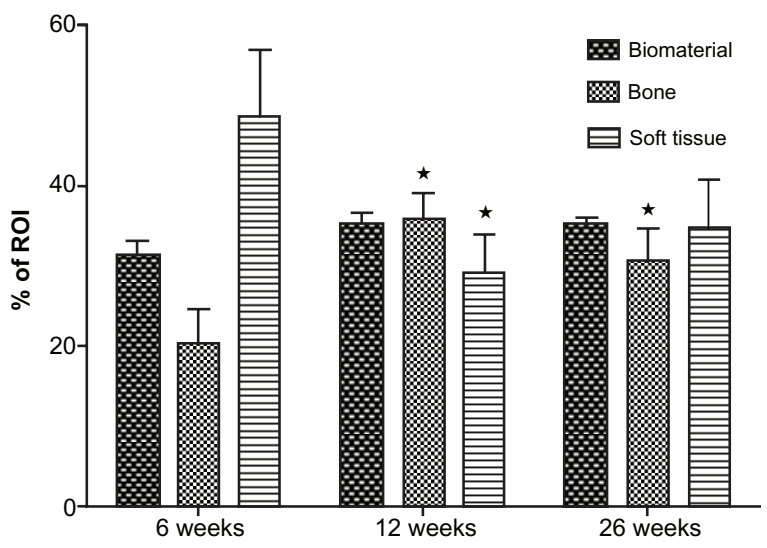

Figure 3 Summary of the histomorphometric data: There is significant increase of the bone area within the first 3 months, while the fraction of the biomaterial remains unaffected over time.

Notes: The fraction of the soft connective tissue shows a marked decrease within the first 12 weeks. Mean value \pm standard error of the mean; analysis of variance; $* P<0.05$ vs 6 weeks.
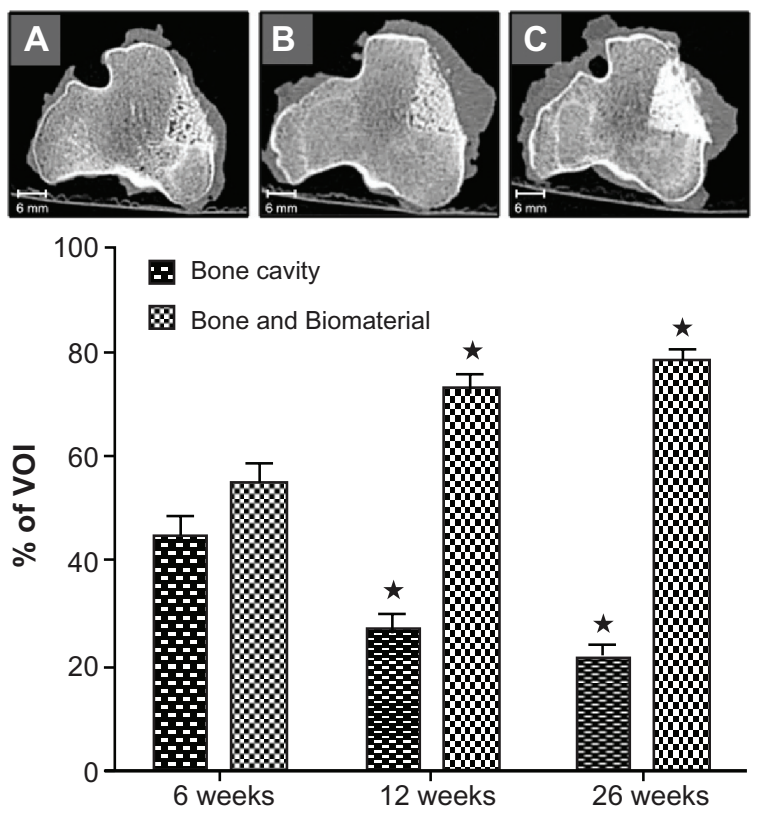

Figure 4 Analysis of the micro-CT data: A decrease of the bone cavity with the simultaneous increase of radiopaque material is significant within the first 3 months, while the size of the cavity is diminishing. Conventional CT images at $6(\mathbf{A}), 12$ (B), and $26(\mathbf{C})$ weeks after the procedure and implantation of the biomaterial confirm these findings.

Notes: Mean value \pm standard error of the mean; analysis of variance; $* P<0.05$ vs 6 weeks.

Abbreviation: $\mathrm{CT}$, computed tomography.

In the present study we investigated the performance of a nanocrystalline hydroxyapatite $\left(\right.$ Nanobone ${ }^{\circledR}$ ) embedded in a porous silica matrix with high porosity and surface area as a bone graft substitute. Application of Nanobone ${ }^{\circledR}$ to a weight-bearing defect of a large animal model has not previously been reported. The control defect showed no signs of a spontaneous bony healing or ingrowth. No signs of overloading were seen nor expected since the parameters of axial loading are well known. ${ }^{23}$ The high-loading capacity of this model can be attributed to the high stiffness of the cortical and trabecular bone in sheep. No deformity or fracture was seen during the study period. The soft tissue healing process was uncomplicated in both the grafted and ungrafted tibiae.

During the entire 26-week period of this study, new bone was formed within the grafted defect with continuous osteoclastic and osteoblastic activity, indicating active bone remodeling. Both the high porosity and the pore size of the bone graft substitute appear to facilitate rapid ingrowth without intervening fibrous tissue. Previous experiments with rats showed that the matrix of Nanobone ${ }^{\circledR}$ was eliminated within a few days. ${ }^{12,24,25}$ Likewise, traces of matrix could not be detected histologically in the present sheep model after the initial study period of 6 weeks. Bone formation occurred 
starting from the defect margins and followed a centripetal direction to the center of the defect. This observation corresponds to the reported deposition of bone from the defect walls toward the implant. ${ }^{26}$

At the bone-HA interface, osteoclastic activity could be observed throughout the entire 26-week experimental period. These findings indicate a high osteoconductivity of nanocrystalline HA. Furthermore, the presence of silicate ions appears to promote the process of bone formation and remodeling at the bone-HA interface. ${ }^{27-29}$

Histomorphometry showed the increase in bone volume inside the defect only within the first 12 weeks. During this time period, the matrix degraded ${ }^{10}$ and was sequentially replaced by connective tissue followed by capillary invasion and differentiation of mesenchymal stem cells into osteoblasts leading to bone formation. ${ }^{30}$ Multinucleated osteoclasts with ruffled borders colonized the HA surface. Degradation of the HA was not observed. Nanobone ${ }^{\circledR}$ is known to induce angiogenesis. ${ }^{31}$ This was confirmed in the present study by the presence of newly formed vessels within the grafted defect. Adequate blood supply is a prerequisite for cellular activity. This finding indicates equilibrium between bone resorption and bone formation during the remodeling process.

Both conventional CT and micro-CT data verified the histological and histomorphometric findings. An increase of radiopaque material within the grafted defect was seen over the first weeks. After 3 months a steady state had been reached. Since the HA was not degraded and remained within the defect, the increased radiopacity in the early time period is fully attributable to newly formed bone.

In the present study there was evidence of both osteoclastic and osteoblastic activity throughout the experimental duration. In contrast to what has been observed with other HA-based bone substitute materials, ${ }^{32}$ the rapid osseointegration of nanocrystalline HA seemed to prevent its complete degradation. At the end of the half-year experimental period, the Nanobone ${ }^{\circledR}$ particles were completely and firmly embedded within newly formed bone without a detectable fibrous interface and with no indication of an adverse host reaction to the material. These factors suggest that degradation of the nanocrystalline material at longer time periods seems unlikely and speak favorably for its clinical use.

\section{Conclusion}

This study demonstrated that Nanobone ${ }^{\circledR}$ is a biocompatible and osteoconductive material in a preclinical, loaded, large-animal model. This study further demonstrated that when implanted into a critical-size defect at the tibial head of sheep, the defect was rapidly filled with new bone that showed signs of vascularization within 3 months after implantation. The matrix binding the HA nanocrystals was quickly degraded, allowing rapid and complete integration of the nanocrystalline HA within the newly formed bone. These properties appear to be promising for the potential use as a bone substitute material in critical bone defects in clinical applications.

\section{Acknowledgments}

The authors thank Artoss $\mathrm{GmbH}$ for supplying the materials, Suzanne Manthey, Annett Wenke, Dorothea Gütschow, and Daniel Wolter for histological technical assistance, and Dr Ing H Martin for performing the micro-CT. The authors are grateful to Visiting Professor Axel Haubold from Decatur, Texas, for his thorough editorial revision of the manuscript.

\section{Disclosure}

The authors report no conflicts of interest in this work.

\section{References}

1. Wippermann BW, Schratt HE, Steeg S, Tscherne H. Complications of spongiosa harvesting of the ilial crest. A retrospective analysis of 1,191 cases. Chirurg. 1997;68(12):1286-1291.

2. Tay BK, Patel VV, Bradford DS. Calcium sulfate- and calcium phosphate-based bone substitutes. Mimicry of the mineral phase of bone. Orthop Clin North Am. 1999;30(4):615-623.

3. Holmes RE, Bucholz RW, Mooney V. Porous hydroxyapatite as a bone-graft substitute in metaphyseal defects. A histometric study. J Bone Joint Surg Am. 1986;68(6):904-911.

4. Jarcho M. Calcium phosphate ceramics as hard tissue prosthetics. Clin Orthop Relat Res. 1981;(157):259-278.

5. Schneiders W, Reinstorf A, Biewener A, et al. In vivo effects of modification of hydroxyapatite/collagen composites with and without chondroitin sulphate on bone remodeling in the sheep tibia. $J$ Orthop Res. 2009;27(1):15-21.

6. Gerike W, Bienengräber V, Henkel KO, et al. The manufacture of synthetic non-sintered and degradable bone grafting substitutes. Folia Morphol (Warsz). 2006;65(1):54-55.

7. Rammelt S, Neumann M, Hanisch U, et al. Osteocalcin enhances bone remodeling around hydroxyapatite/collagen composites. J Biomed Mater Res A. 2005;73(3):284-294.

8. Henkel KO, Gerber T, Lenz S, Gundlach KK, Bienengräber V. Macroscopical, histological, and morphometric studies of porous bonereplacement materials in minipigs 8 months after implantation. Oral Surg Oral Med Oral Pathol Oral Radiol Endod. 2006;102(5):606-613.

9. Canullo L, Dellavia C. Sinus lift using a nanocrystalline hydroxyapatite silica gel in severely resorbed maxillae: histological preliminary study. Clin Implant Dent Relat Res. 2009;11 Suppl 1:e7-e13.

10. Götz W, Gerber T, Michel B, Lossdorfer S, Henkel KO, Heinemann F. Immunohistochemical characterization of nanocrystalline hydroxyapatite silica gel (NanoBone ${ }^{\circledR}$ ) osteogenesis: a study on biopsies from human jaws. Clin Oral Implants Res. 2008;19(10):1016-1026.

11. Gerber T, Holzhüter G, Götz W, Bienengräber V, Henkel KO, Rumpel E. Nanostructuring of biomaterials - a pathway to bone grafting substitute. Eur J Trauma. 2006;32(2):132-140. 
12. Webster TJ, Ahn ES. Nanostructured biomaterials for tissue engineering bone. Adv Biochem Eng Biotechnol. 2007;103:275-308.

13. Henkel KO, Gerber T, Dorfling P, et al. Stimulating regeneration of bone defects by implantation of bioceramics and autologous osteoblast transplantation. Mund Kiefer Gesichtschir. 2002;6(2):59-65.

14. Traykova T, Bötcher R, Neumann HG, Henkel KO, Bienengräber V, Gerber T. Silica/calcium phosphate sol-gel derived bone grafting material - from animal tests to first clinical experience. Key Eng Mat. 2004;254-256:679-682.

15. Henkel KO, Gerber T, Dorfling P, Gundlach KK, Bienengräber V. Repair of bone defects by applying biomatrices with and without autologous osteoblasts. J Craniomaxillofac Surg. 2005;33(1):45-49.

16. Ignatius $\mathrm{A}$, Unterricker $\mathrm{K}$, Wenger $\mathrm{K}$, et al. A new composite made of polyurethane and glass ceramic in a loaded implant model: a biomechanical and histological analysis. J Mater Sci Mater Med. 1997; 8(12):753-756.

17. Ignatius $\mathrm{AA}$, Betz $\mathrm{O}$, Augat $\mathrm{P}$, Claes LE. In vivo investigations on composites made of resorbable ceramics and poly(lactide) used as bone graft substitutes. J Biomed Mater Res. 2001;58(6):701-709.

18. Ignatius A, Peraus $\mathrm{M}$, Schorlemmer S, et al. Osseointegration of alumina with a bioactive coating under load-bearing and unloaded conditions. Biomaterials. 2005;26(15):2325-2332.

19. Rammelt S, Manthey S, Zwipp H. Immunohistochemical characterization of the bone-implant interface on MMA-embedded cutting and grinding sections. Eur Cell Mater. 2004;7(Suppl 2):82.

20. Rammelt S, Corbeil D, Manthey S, Zwipp H, Hanisch U. Immunohistochemical in situ characterization of orthopedic implants on polymethyl metacrylate embedded cutting and grinding sections. J Biomed Mater Res A. 2007;83(2):313-322.

21. Tamai N, Myoui A, Tomita T, et al. Novel hydroxyapatite ceramics with an interconnective porous structure exhibit superior osteoconduction in vivo. J Biomed Mater Res. 2002;59(1):110-117.
22. Hulbert SF, Morrison SJ, Klawitter JJ. Tissue reaction to three ceramics of porous and non-porous structures. J Biomed Mater Res. 1972;6(5): 347-374.

23. Simon U, Augat P, Ignatius A, Claes L. Influence of the stiffness of bone defect implants on the mechanical conditions at the interface - a finite element analysis with contact. J Biomech. 2003;36(8):1079-1086.

24. Xu W, Holzhuter G, Sorg H, et al. Early matrix change of a nanostructured bone grafting substitute in the rat. J Biomed Mater Res B Appl Biomater. 2009;91(2):692-699.

25. Xu W, Ganz C, Weber U, et al. Evaluation of injectable silica-embedded nanohydroxyapatite bone substitute in a rat tibia defect model. Int $J$ Nanomedicine. 2011;6:1543-1552.

26. Hing KA, Best SM, Tanner KE, Bonfield W, Revell PA. Biomechanical assessment of bone ingrowth in porous hydroxyapatite. J Mater Sci Mater Med. 1997;8(12):731-736.

27. Porter AE, Patel N, Skepper JN, Best SM, Bonfield W. Effect of sintered silicate-substituted hydroxyapatite on remodelling processes at the bone-implant interface. Biomaterials. 2004;25(16):3303-3314.

28. Magallanes-Perdomo M, De Aza AH, Mateus AY, et al. In vitro study of the proliferation and growth of human bone marrow cells on apatitewollastonite-2M glass ceramics. Acta Biomater. 2010;6(6): 2254-2263.

29. Patel N, Best SM, Bonfield W, et al. A comparative study on the in vivo behavior of hydroxyapatite and silicon substituted hydroxyapatite granules. J Mater Sci Mater Med. 2002;13(12):1199-1206.

30. Burchardt H. The biology of bone graft repair. Clin Orthop Relat Res. 1983;(174):28-42.

31. Abshagen K, Schrodi I, Gerber T, Vollmar B. In vivo analysis of biocompatibility and vascularization of the synthetic bone grafting substitute NanoBone. J Biomed Mater Res A. 2009;91(2):557-566.

32. Spies CK, Schnurer S, Gotterbarm T, Breusch S. The efficacy of Biobon and Ostim within metaphyseal defects using the Gottinger Minipig. Arch Orthop Trauma Surg. 2009;129(7):979-988.
International Journal of Nanomedicine

\section{Publish your work in this journal}

The International Journal of Nanomedicine is an international, peerreviewed journal focusing on the application of nanotechnology in diagnostics, therapeutics, and drug delivery systems throughout the biomedical field. This journal is indexed on PubMed Central,

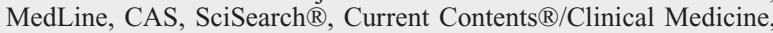

\section{Dovepress}

Journal Citation Reports/Science Edition, EMBase, Scopus and the Elsevier Bibliographic databases. The manuscript management system is completely online and includes a very quick and fair peer-review system, which is all easy to use. Visit http://www.dovepress.com/ testimonials.php to read real quotes from published authors. 